\title{
Comprehensive Surveillance Needed to Contain COVID-19
}

\author{
Saurabh RamBihariLal Shrivastava MD and Prateek Saurabh Shrivastava MD
}

The coronavirus pandemic has shaken public health authorities around the world and raised questions about health sector preparedness in responding to infectious disease outbreaks. As of December 7, 2020, more than 66 million cases had been reported globally, and more than 1.5 million people have died from the infection in 219 nations and territories.[1,2] The hard lesson is that preparedness must be urgently improved, including case detection through better surveillance; improved laboratory diagnostic facilities; more and better-equipped isolation wards and treatment facilities; increased supply and better distribution of personal protective equipment; more effective risk communication; and adoption of community engagement action plans. $[1,3]$

\section{Preparedness must be urgently improved, including case detection through better surveillance}

Surveillance is key. Strengthening surveillance in China and elsewhere has been acknowledged as a prime component for effective response to minimize the number of cases and prevent further loss of life.[4] This has proven true in other scenarios as well, such as the 2014 Ebola outbreak in West Africa, where stronger surveillance proved to be a main factor for effective containment.[5]

Case detection and confirmation testing, as well as contact tracing, must include effective surveillance at all international points of entry-airports, border crossings and maritime ports.

Turning for a moment to our own country, India: we can assume significant under-reporting of cases, given the country's population density and other sociodemographic factors that, taken together, favor rapid COVID-19 transmission. We believe this may be due to deficient surveillance compounded by insufficient availability of testing facilities. In particular, comprehensive surveillance is hobbled by lack of diagnostic kits in laboratories and community settings. This precludes more realistic caseload estimates and thus hinders more precise forecasts of logistic requirements. It is paramount at this stage to increase laboratory capacities and to include private facilities in the panel of approved laboratories where this has not already been done.[6]

Returning to the global community: a worldwide surveillance system is already in a place capable of collecting and organizing data on disease magnitude and distribution.[3] Many countries have surveillance systems and/or electronic data collection mechanisms which transmit information daily from WHO regional offices to its Geneva headquarters.[3,4] This requires countries to adhere to established case definitions-suspect, probable and confirmed-for reporting COVID-19 infections,[3] providing data in a prescribed format in order to align global estimates. According to WHO surveillance guidelines, these data would ideally include details on the number of cases, deaths, laboratory tests performed, positive results and number of contacts being monitored. Further, it is recommended that each nation provide clinical-epidemiological travel histories of any new cases within two days of confirmation. Member states should use WHO-established categories for classifying transmission status-no cases, sporadic cases, clusters or community transmission-in order to strengthen global surveillance, enable risk assessment at various levels, assist in monitoring disease caseloads and provide evidence for national health authorities to mount a better public health response.[4]

However, it is important to acknowledge that such comprehensive surveillance is only efficient and effective in those nations that had viable health systems prior to the outbreak. Both WHO recommendations and our own would prove impractical for strengthening surveillance in nations facing system-wide challenges such as fragmented healthcare delivery; access disparities; scarcity of trained personnel; and financial or technological constraints. These systemic issues make it extremely difficult to perform risk assessments, provide timely detection and monitor overall caseload.

In those countries with weaker surveillance systems, strategies should be implemented to: ensure the general population is at least informed about COVID-19 and how it is transmitted; encourage selfquarantine; and improve health-seeking behavior by symptomatic individuals. If the need arises based on caseload, active surveillance can be adopted with the help of outreach workers, trained as part of a community engagement action plan. In order to gain extra leverage and time to improve readiness and reduce transmission, many nations have imposed lockdowns, but these are passive interventions: once lockdown is over, cases will rise once again-as is happening currently in many countries. Lockdowns can be used effectively to actively search for cases in the community, while managing suspect or confirmed cases according to established protocols.

In essence, complacency is not an option for any country and surveillance has proven integral to preparedness, prevention and control of the COVID-19 pandemic. As nations review their health systems' viability, ample attention should be paid to implementing effective surveillance activities, with sufficient support for public health authorities. Our preparedness now-and in the face of future infectious threats-depends on it. -1 -

\section{References}

1. World Health Organization. Statement on the second meeting of the International Health Regulations (2005) Emergency Committee regarding the outbreak of novel coronavirus (2019-nCoV) [Internet]. Geneva: World Health Organization; 2020 Jan 30 [cited 2020 Feb 28]. Available at: https://www.who .int/news-room/detail/30-01-2020-statement-on-the-second-meeting-of-the -international-health-regulations-(2005)-emergency-committee-regarding-the -outbreak-of-novel-coronavirus-(2019-ncov)

2. World Health Organization. Coronavirus (COVID-19) Dashboard [Internet]. Geneva: World Health Organization; 2020 Dec 7 [cited 2020 Dec 7]. Available at: https://covid19.who.int

3. World Health Organization. 2019 Novel Coronavirus (2019-nCoV): Strategic preparedness and response plan. Geneva: World Health Organization Press; 2020. p. 1-8

4. World Health Organization. Global Surveillance for human infection with novel coronavirus (2019-nCoV) - Interim guidance. Geneva: World Health Organization Press; 2020. p. 1-3.

5. Shrivastava SR, Shrivastava PS, Ramasamy J. Lessons learnt from the 2014 Ebola outbreak in West Africa. J Res Med Sci. 2015 Jan;20(1):107-8.

6. Indian Council of Medical Research [Internet]. New Delhi: Indian Council of Medical Research; c2020. COVID-19. Information for Testing Laboratories; [cited 2020 Oct 12]; [about 3 p.]. Available at: https://www.icmr.gov.in/ctestlab.html

Submitted: February 28, 2020

Approved for publication: November 12, 2020

Disclosures: None

Correspondence: drshrishri2008@gmail.com

https://doi.org/10.37757/MR2021.V23.N1.1 